\title{
Communicative Arabic and Structure of Its Philosophical Teaching
} اللغة العربية الاتصالية وهيكل تعليمها الفلسفي

\section{Muhammad Rusydi ${ }^{1}$}

${ }^{1}$ Institut Agama Islam Negeri Bone, Watampone, Indonesia. E-mail: rusydi.stainwtp@yahoo.com

\begin{tabular}{l} 
ARTICLE INFO \\
\hline Keywords: \\
Communicative Arabic; \\
Teaching; Philosophical \\
Framework \\
How to cite: \\
Rusydi, M. (2018). \\
Communicative Arabic \\
and Structure of Its \\
Philosophical Teaching, \\
4(1), 46-52. \\
DoI: \\
http://dx.doi.org/10.31332/ \\
lkw.v4i1.747 \\
\hline
\end{tabular}

\begin{abstract}
This article orients to expose the philosophical frame of communicative Arabic teaching. Communicative Arabic teaching has a strong philosophical framework because this modern teaching approach directs the Arabic language to its original position as a tool to communicate. In this context, the ontology dealing with the essence of Arabic teaching as a pedagogical reality, the epistemology dealing with its procedural framework and the ontology dealing with its value framework see that communicative Arabic teaching should be developed with a suitable educational philosophy reinforcing the Arabic to its functionalcommunicative framework. This article, finally, presents an alternative analysis to perceive communicative Arabic teaching.
\end{abstract}

تعليم اللغة العربية يشير إلى تقدم سريع في عدة جوانب من المدخل كلهيل الأكسيوماتي والطريقة كلهيكل الإجرائي والأسلوب كلهيكل التطبيقي. التقدم يكون دليل في إرتفاع أهمية اللغة العربية في موقفها كلغة اتصالية في العالم. في هذا الموقف، اللغة عامة واللغة العربية خاصة قد أشارها عبد العزيز إبراهيم الأصيلي كوسيلة استخدمها الإنسان في السياقات الإتصالية المختلفة وتتضح فيها عدة طرق تعليم اللغة العربية مثل الطريقة المباشرة والطريقة الطبيعية والطريقة الواقعية وهلم جرا (الأصيلي، ب . . r).

موقف اللغة العربية كمجال العلوم لا ينفصل من نموذج العلوم وتطورها. في تطور العلوم، معظم الخبراء يفرقون بين العلوم الطبيعية والعلوم الإنسانية من خلال مواضيعها بخصيصتها المعينة. مواضيع العلوم الطبيعية تتخصص بالأشياء الثابتة ومواضيع العلوم الإنسانية تتخصص بالأشياء غير ثابتة. ولذلك، قد رأى ويلحلم ديلتهى الفرق بين تعلم العلوم الطبيعية وتعلم العلوم الإنسانية من الناحية الإيبستمولوجية (Epistemology) التي تتفرق عند معظم الخبراء الذين يرون من الناحية الأنتولوجية (Aksiology). في رؤية ويلحلم ديلتي، تعلم العلوم الطبيعية يطلب انفصال شخصية الأشخاص المشتركين فيه لنيل الموضوعية في فهم الظواهر الطبيعية وفي العكس تعلم العلوم الإنسانية يطلب 
اشتراك شخصية الأشخاص المشتركين فيه لنيل الموضوعية في فهم الظواهر الإنسانية الواقعة (Ilyas Supena ، . (r... O

تعليم اللغة العربية الاتصالية لا ينفصل من الهيكل الفلسفي في فهم الظواهر الطبيعية والظواهر الإنسانية بسبب تطبيقه يطلب اتحاد تعليم اللغة الذي يؤدي إلى فهم استخدام اللغة العربية كلغة اتصالية والتعليم عن اللغة الذي يؤدي إلى فهم اللغة العربية من عناصرها وعلومها ومهارتا بخصيصتها المختصة. والهيكل الفلسفي في هذا النشاط سوف يوجه عملية تعليم اللغة العربية في إطاره الوظيفي والاتصالي. هذا البحث هو تطوير ما كتب الباحث في رسالة الدكتوراه بجامعة مولانا مالك ابراهيم بموضوع "أفكار أزهار أرشلد التجلديدية المحلية في تجديد إيبستمولوجيا تعليم اللغة العربية وتطبيقها في الجامعات الإسلامية الحكومية بسولاويسي الجنوبية" واختلاف.

Y. البحث اللغة العربية الإتصالية وتعليمها

في موقفها كمدخل جديد في التعلم الحديث، اللغة العربية الإتصالية تتأسس على وظيفة اللغة في حياة

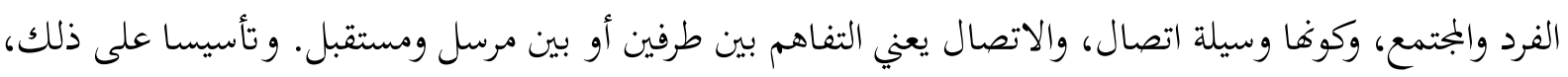
يمكن القول إن الاتصال اللغوي أكثر من شكل فمنه الاتصال اللفظي والمنطوق. ومنه الاتصال اللفظي المكتوب، ولكل منهما موضوعاته وأهدافه وشروطه، فالإرسال اللفظي يقتضي وضوح الصوت وتنويع نبراته وتدعيمه بالوسائل المصاحبة كتقاسيم الوجه وحركة اليدين أو غير ذلك مما يقتضيه سياق الخطاب. كذلك للإرسال المكتوب شروط يجب توافرها فيه كصحة الرسم وتنظيم الفقرات وعلامة الترقيم التي تدل على أساليب التعبير الصوتي عن المعاني المقصودة (علي عطية، V... V) أما بدرالدين أبو صالح يري تعليم اللغة العربية الاتصالية كعملية التعليم الذي يبتدأ من حفظ المفرادات المناسبة بالبيئة الاتصالية ويتبعه إتقان التعبيرات والأساليب الشفوية العربية في سياقات الاتصال المختلفة (أبو صالح) هذا الرأي يشير إلى نموذجتعليم اللغة العبية الاتصالية المتخصص في طاقة الاتصال الشفوي بحفظ المفردات الشائعة بأكثر مايمكن وتطبيقها بتدريبات الأنماط الشفوية مثل ما يلي:

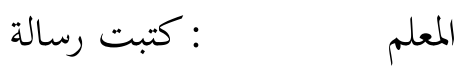
المتعلم 1 : : كتبت رسالة أمعلم

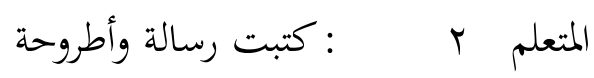
في إجراء بتدريبات الأنماط الشفوية، المرسل والمستقبل يطوران استخدام المفردات المعروفة في العلاقات الاتصالية. وفي ضمان لبعد الاتصالي في تعليم اللغة العربية الاتصالية، هناك العديد من الشروط لا بد من تحقيقها. 
والشروط توجد في المرسل والرسالة اللغوية وقناة الإرسال والمستقبل والتغذية الراجعة وبيئة الاتصال كعناصر الاتصال

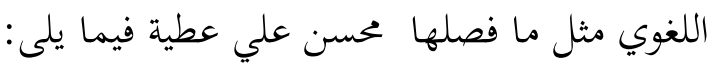

أ. الشروط في المرسل فهي أن يكون ذا معرفة تامة بموضوع الرسالة وما تتصل بها، وأن يكون ذا خبرة وبحربة تؤهله

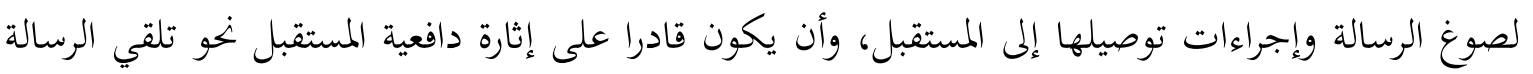
وتفاعله معها طوال عملية الاتصال، وأن يكون عارفا بطرائق التوصيل المختلفة، وغير ذلك.

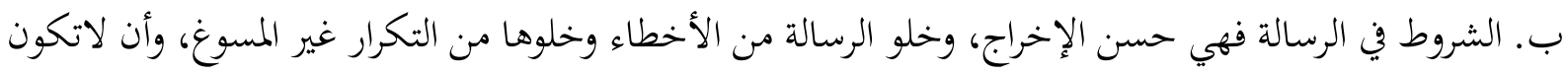

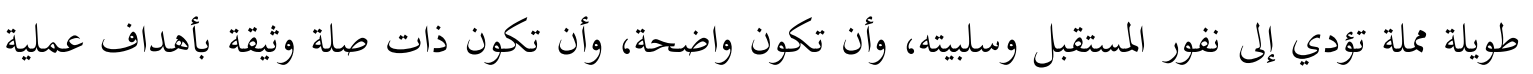
الاتصال، وغير ذلك.

ج. الشروط في قنات الاتصال فهي أن ترتبط بمحتوى الرسالة، وأن تلائم قدرات المتعلمين وتحصيلهم اللغوي، وأن يكون أسلوب الغرض ملائما، وغير ذلك.

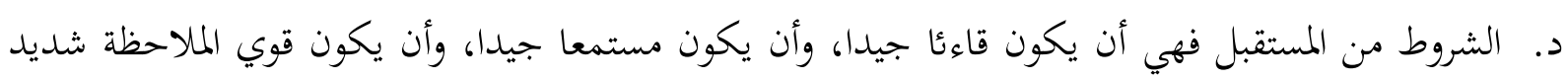
الانتباه، وأن يكون راغبا في موضوع الرسالة، وغير ذلك.

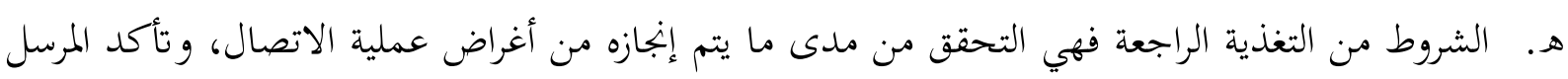
من أن رسالته حققت مأراده منها، وغير ذلك.

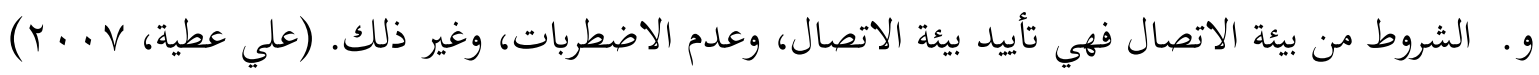
بالاضافة إلى ماتم نقله من محسن علي عطية سابقا، قد زاد إمام معروف أن تعليم اللغة العربية الاتصالية

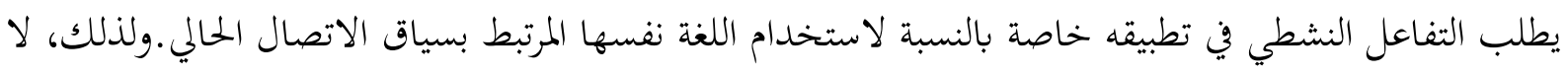

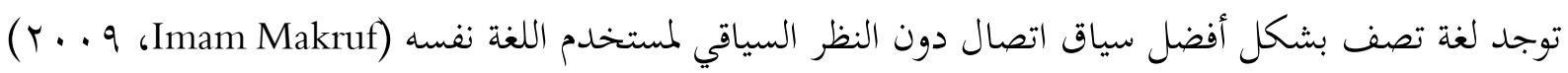

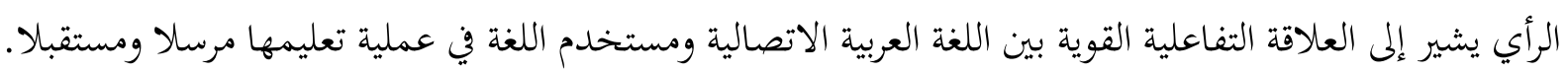

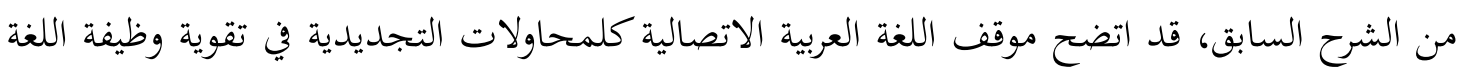
العربية في وظيفتها الاتصالية بعدة شروط تشيرها عناصر الاتصال اللغوي من المرسل والرسالة اللغوية وقناة الإرسال والمستقبل والتغذية الراجعة وبيئة الاتصال. فلسنة تعليم اللغة العربية

فلسفة تعليم اللغة العربية هي الهيكل الفلسفي عن اللغة العربية وتعليمها من أنتولوجيا (Ontology)

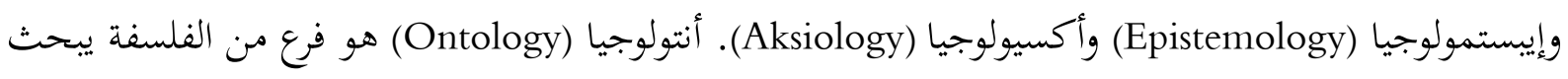

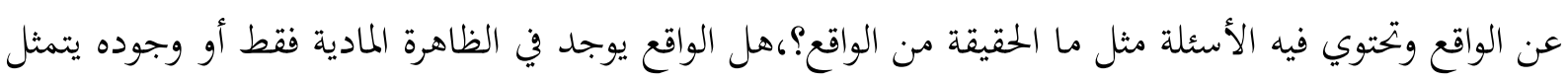

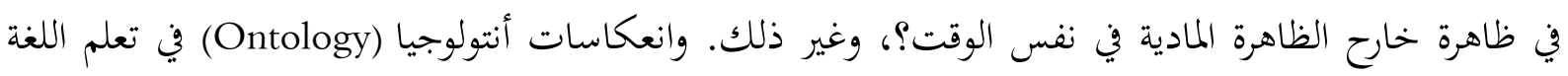


العربية هي نظرة فلسفية لواقع التعلم العربي الشامل كعملية تتضمن فيه مكونات تعلمية مختلفة بأدوار مختلفة مثل دور المعلم، ودور المتعلمين، ودور المواد التعليمية، ودور الأهداف التعليمية، ودور الأنشطة التعليمية. ولذلك، أنتولوجيا (Ontology) تعليم اللغة العربية هو انعكاس فلسفي للواقع الذي يجيط بتعليمها المبنى من التخطيط والتنفيذ والتقييم. وقال جيجيف سومارنا (Cecep Sumarna) أن فهم الواقع في الإطار الفلسفي يؤدي إلى تقدم الناس في

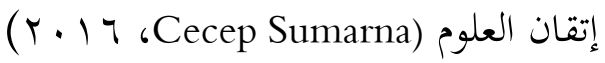
إيبستمولوجيا (epistemology) تعليم اللغة العربية لا ينفصل محا قدم Dagobert D. Runes من التعريف وهو أن إيستمولوجيا (epistemology) فرع من فروع الفلسفة يناقش مصادر المعرفة، وتركيبها، ومنهجها، وصحتها (19 و ( D. Runes)

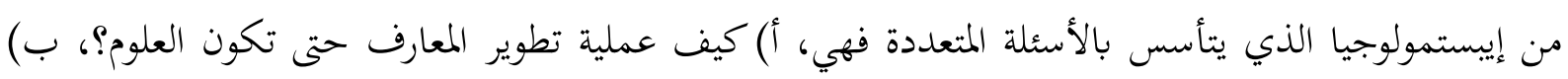
وكيف إجراءات تطوير المعارف حتى تكون العلوم؟، ج) وماهي الأشياء المهمة لابدلنا أن هتم بها لنيل المعارف الصحيحة؟، د) وماهو الحق نفسه؟ وما معاييره؟، هـ) وماهي المنهجيات والوسائل التي تساعدنا لنيل المعارف كالعلوم؟ († ( . Amtsal Bakhtiar) وتعليم اللغة العربية كجزء من عملية تطوير العلوم لابد أن يهتم بإيبستمولوجيا (epistemology) في تطبيقه. وقد قال محبيب عبد الوهاب أن البحث عن إيبستمولوجيا (epistemology) تعليم اللغة العربية يعني البحث عن موقف اللغة العربية كالعلوم نفسها. وبالنسبة لأهمية إيبستمولوجيا (epistemology) تعليم اللغة العربية، قد زادمحييب عبد الوهاب أن البحث عن إيبستمولوجيا مهم بسبب الاسباب فهي أ) وظيفة العلوم الرئيسية هي الشرح. وفي تطبيقه، تعليم اللغة العربية كجزء من العلوم لابد أن يستطيع لشرح مكانته و موضوعه. ولذلك، قد اتضح أهمية إيبستمولوجيا (epistemology) تعليم اللغة العربية في إعطاء الشرح النظري والتطبيقي عنتعليم اللغة العربية في تطبيقه وتطويره، ب) وصفة العلوم تراكمية ومنطقية. من صفة العلوم نعرف أها تتطور متدرجة ومتصلة. كجزء من العلوم،

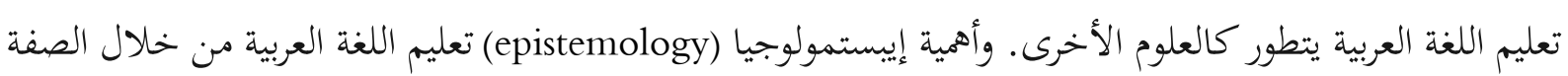
هي تصوير إلي أي مدى تطور تعليم اللغة العربية، ج) ابتاهات التطوير العلمي لتعليم اللغة العربية لابد أن تتطور في الإطار الإيبستمولوجي الصحيح. من الإبحاهات، نعرف أهمية إيبستمولوجيا تعليم اللغة العربية لتوجيه إجراء تعليم

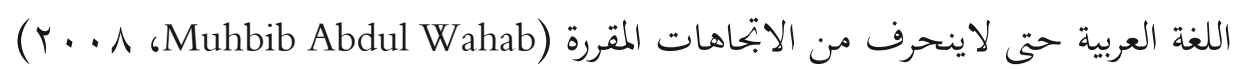
أكسيولوجيا (Aksiology) تعليم اللغة العربية هو فرع من الفلسفة يبحث عن الإطار الفلسفي في اللغة

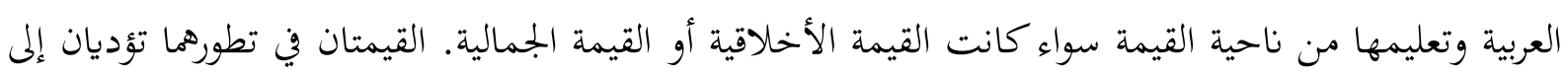
ابتحاهات تعليم اللغة العربية ومنافعه في نفس النشاط. وابتحاهات تعليم اللغة العربية قد فصلها محمود يونس بأربعة

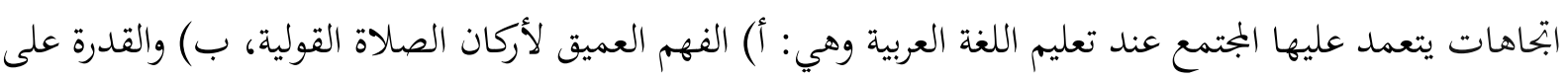

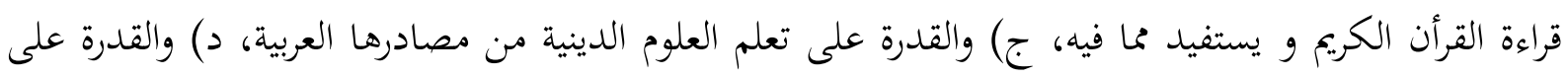


الإنشاء الشفهي والتحريري باللغة العربية خلال العلاقة والمعاملة بين المسلمين لأن اللغة العربية لغة المسلمين في أنحاء

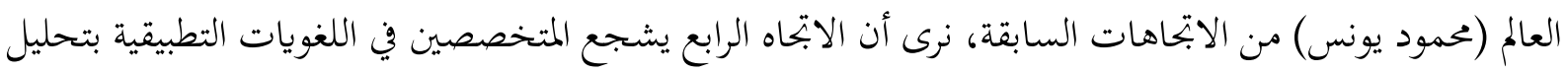

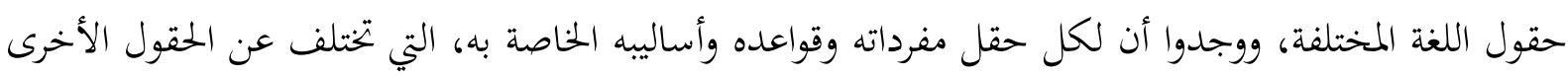
وهذا الإتحاه يتعمق فيه تعليم اللغة العربية الاتصالية. تأسيسا على ما فصل محمود يونس من الاتحاهات كجزع من أكسيولوجيا (Aksiology) تعليم اللغة العربية، قدم أجف حيرماوان إلى ابحاهات عديدة منها:

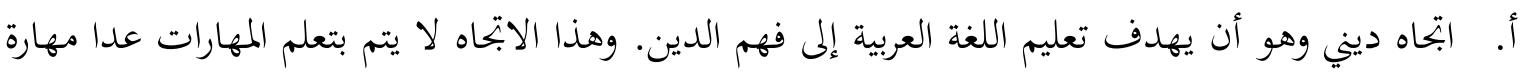
القراءة و الترجمة

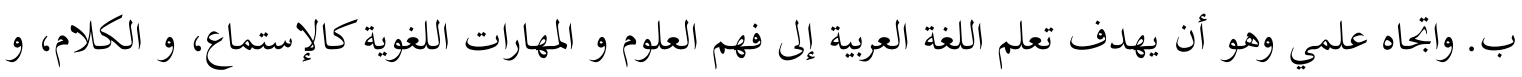
القراءة، و الكتابة ج. واتجاه فكري و اقتصادي و هو أن يهدف تعليم اللغة العربية إلى فهم اللغة العربية و القدرة على استخدامها

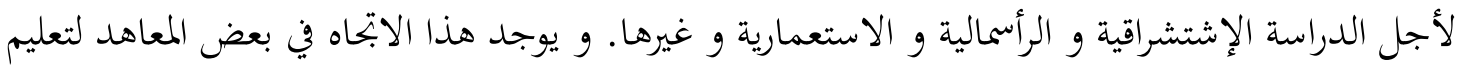

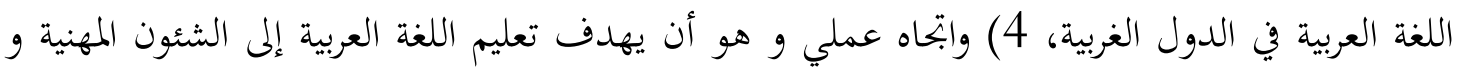

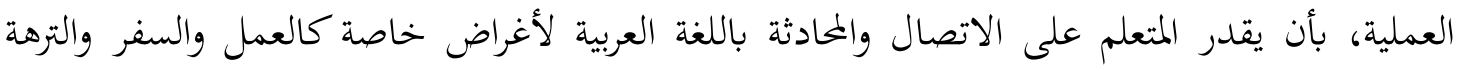
والتجارة (r. 11،Acep Hermawan) من الشرح السابق، قد اتضح أن فلسفة تعليم اللغة العربية تضع تعليم اللغة العربية في الهيكل الفلسي

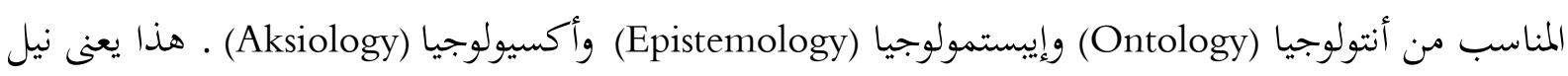

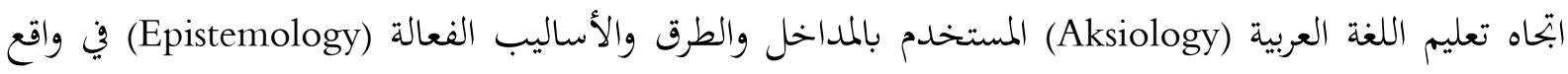
تعليم اللغة العربية كعلم (Ontology).

$$
\text { الهيكل الفلسني في تعليم اللغة العببية الاتصالية }
$$

تعليم اللغة العربية كجزء من العلوم الإنسانية لابد من استفادة النموذج أن تعلم العلوم الإنسانية من ناحيته

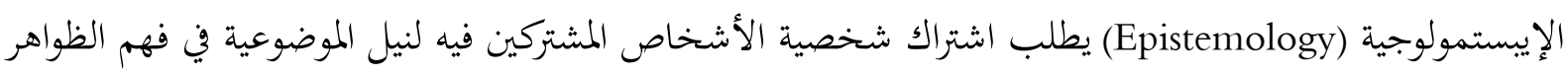

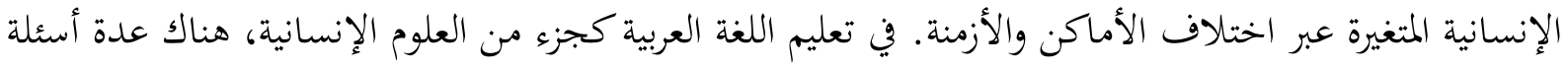

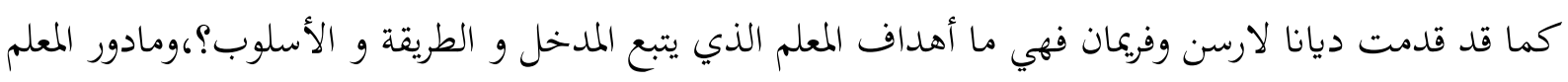

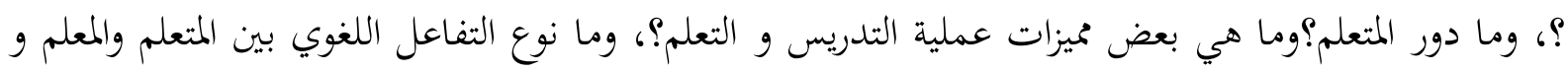

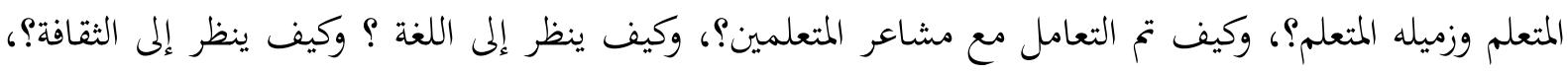

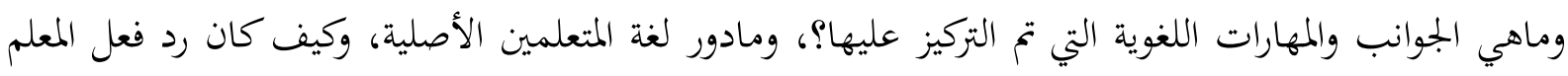

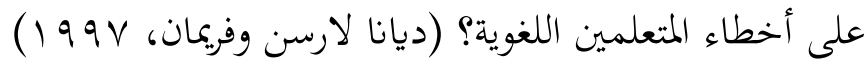


في الهيكل الفلسفي، تعليم اللغة العربية الاتصالية يمكن فهمه من ثلاثة جوانب فهي أنتولوجيا وإيبستمولوجيا وأكسيولوجيا. هذه الجوانب الثلاثة تمتلك العلاقة التفاعلية في شرح تعليم اللغة الاتصالية وهيكل تعليمها الفلسفي. وهذه هي الخصيصة في التفكير الفلسفي الذي يطلب العلاقات من موضوعيات الفكرة المتعددة حتى تتضح فيها الأجوبة الفلسفية الكاملة من أنتولوجيا وإيبستمولوجيا وأكسيولوجيا. في أنتولوجيا (Ontology)، تعليم اللغة العربية الاتصالية لايكفي فهمه كعملية التعليم اللغوي من علوم اللغة العربية وعناصرها ومهارتحا. في هذا النشاط، الوظائف الاتصالية من اللغة العربية يجب تأكيدها من خلال تعليم اللغة العربية وعناصرها ومهارها. في شرح أكسيولوجيا (Aksiology) تعليم اللغة العربية الاتصالية من الخصيصة، قد قدم سوبياكتو وسيري أوتارى (Subiyakto and Sri Utari) الخصائص المختصة في فهم أكسيولوجيا تعليم اللغة العربية الاتصالية فهي: أ) عملية التعليم يشير إلى النشاط الواقعي لتشجيع المتعلمين في تعلم اللغة العربية كلغة اتصالية، ب) والأنشطة اللغوية تتجه إلى الواجبات الأتصالية لتشجيع المتعلمين في تعلم اللغة العربية كلغة اتصالية، ج) والمواد التعليمية وخطتها المطورة تتأسس على تحليل الحاجات من المتعلمين، د) وعملية التعليم تتأسس على المتعلمين، هـ) ومعلم اللغة العربية يقوم بدور كمدير التعليم ومحلله ومشجعه لتشجيع المتعلمين في تعلم اللغة العربية كلغة اتصالية، و) ودور المواد التعليمية هو تأييد إجراء تعليم اللغة العربية النشاط (Subiyakto and Sri Utari)، ب99 1 ) في إيبستمولوجيا (Epistemology)، تعليم اللغة العربية الاتصالية لاينفصل من أربع مكونات إيبستمولوجيا فهي مصدر تعليم اللغة العربية وتركيبه ومنهجيته وصحته. في المصدر، تعليم تعليم اللغة العربية (Epistemology) الاتصالية يحتاج إلى التطوير المستمر ـ في أول تعليمها، مصدر تعليم اللغة العربية لا ينفصل من المصادر النصية وتعليم اللغة العربية الاتصالية جاء بعدة مصادر تطويرية يستفيد من الكون الداخلي (بالوهبة البديهية بالإلمام والتحليل القياسي بالعقل) والكون الخارجي (الأشياء الواقعية بالحس) كتطبيق من بتحيد مصدر تعليم اللغة العربية (رشدي،

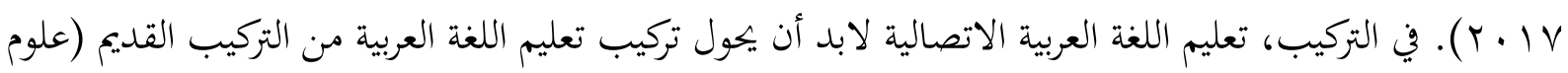
اللغة العربية وعناصرها ومهارتما) الى التركيب الجديد والخاص في تعليم اللغة العربية الاتصالي (ارتفاع حفظ المفردات وتطوير علاقة المفردات السياقية وتقوية الحماسة الداخلية). في المنهجية، تعليم اللغة العربية الاتصالية أن يستفيد من من المدخل الاتصالى كلهيكل الأكسيوماتي (Aksiomatic) وتتبعه طرق تعليم اللغة العربية الاتصالية المعاصرة مثل الطريقة الإيحائية وطريقة تعليم اللغة الجماعي والطريقة الصامتة مع الأساليب المناسبة. وفي النهاية، صحة تعليم اللغة العربية الاتصالية تتصور من عدة نظريات مثل نظرية المراسلات ونظرية التماسك والنظرية البارغماتية. في أكسيولوجيا (Aksiology)، تعليم اللغة العربية يتأسس على القيم الأخلاقية والقيم الجمالية. وقال خالد محمود محمد عرفان تعليم اللغة العربية لابد من توجيهه في الاتحاهات السلوكية والاتحاهات اللغوية والاتحاهات

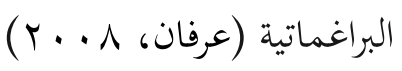


من الشرح السابق، قد اتضح الهيكل الفلسفي في تعليم اللغة العربية الاتصالي يستفيد كثيرا من المحاولات التجديدية الفلسفية في تقوية وظيفة اللغة العربية الاتصالية أنتولوجيا وإيبستمولوجيا وأكسيولوجيا. r.

الهيكل الفلسفي لتعليم اللغة العربية الاتصالية يشير إلى كيفية تأسيس عناصر الاتصال اللغوي من المرسل والرسالة اللغوية وقناة الإرسال والمستقبل والتغذية الراجعة وبيئة الاتصال في عناصر الفلسفة الثلاثة فهي أنتولوجيا

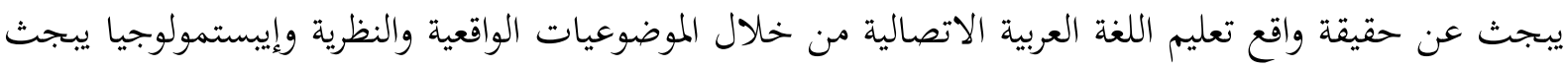

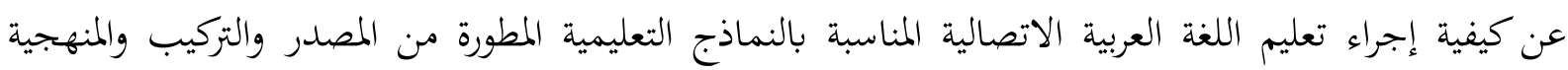
والصحة وأكسيولوجيا يبجث عن قيم تعليم اللغة العبية الاتصالية أخلاقية وجمالية وتتمثل فيها اتبحاهات تعليمها. المراجع

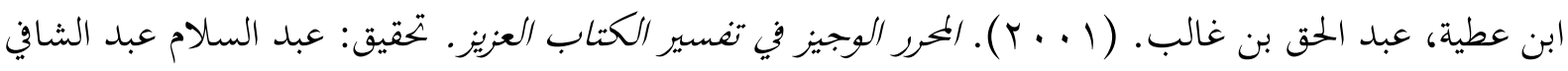
محمد، طا؛ بيروت: دار الكتب العلمية. أبو صالح، بدرالدين. (دون سنة). المدخل إلى اللغة العربية. بيروت: دار الشرق العربي.

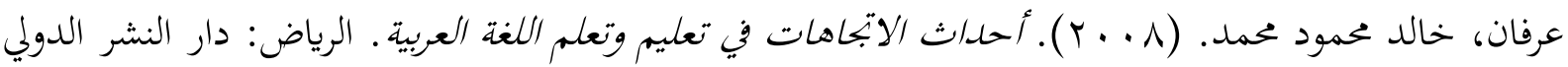
للنشر والتوزيع.

لارسن، ديانا، وفريمان. (997 (1). تربمة عائشة موسى السعيد، أساليب ومبادئ في تدريس اللغة. الرياض: جامعة

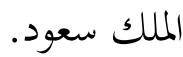

الأصيلي، عبد العزيز إبراهيم. (r . . r). طرائق تعليم اللغة العربية للناطقين بلغة أخرى. الرياض: جامعة الإمام محمد إبن سعود الإسلامية.

عطية، محسن علي. (V . . †). مهارات الاتصال اللغوي وتعليمها ـ عمان: دار المناهج للنشر والتوزيع.

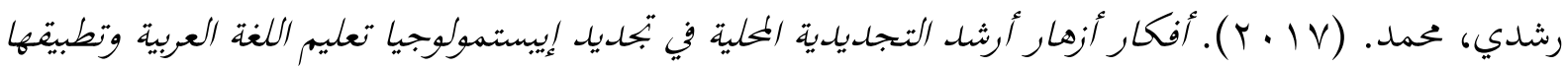

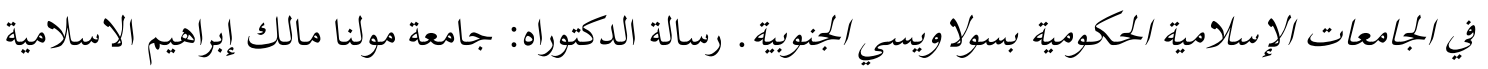
الحكومية مالانج.

Abdul Wahab, Muhbib. (2008). Epistemologi dan Metodologi Pembelajaran Bahasa Arab. Jakarta: UIN Jakarta Press.

Bakhtiar, Amsal. (2014). Filsafat Ilmu. Jakarta: PT. Raja Grafindo Persada.

Hermawan, Acep. (2011). Metodologi Pembelajaran Bahasa Arab. Bandung: PT. Remaja Rosdakarya.

Makruf, Imam. (2009). Strategi Pembelajaran Bahasa Arab Aktif. Semarang: Need's Press 
Runes, Dagobert D. (1963). Dictionary of Phylosophy. New Jersey: Little Field Adams \& Co.

Subiyakto, Sri Utari. (1993). Metodologi Pengajaran Bahasa. Jakarta: Gramedia.

Sumarna, Cecep. (2016). Filsafat Pengetahuan. Bandung: PT. Remaja Rosdakarya.

Supena, Ilyas. (2015). Rekonstruksi Epistemologi Ilmu-Ilmu Keislaman. Yogyakarta: Penerbit Ombak. 